\title{
Slow maxillary expansion: a comparison between banded and bonded appliances
}

\author{
Katarina Mossaz-Joëlson and Claude F Mossaz \\ Department of Orthodontics, Dental School, University of Geneva, Switzerland
}

SUMMARY Various investigations have mentioned the use of a bonded maxillary expansion appliance. It was postulated that a full coverage of the occlusal surfaces by acrylic would remove interferences during the lateral displacement of the two maxillary bones and would lessen the resistance to maxillary expansion. The first objective of this study was to compare two appliances, a bonded and a banded Minne expander using a continuous force of two pounds, which would produce a slow maxillary expansion. The second objective was to evaluate the ratio between the skeletal and dental response to slow maxillary expansion, and to compare these results to those obtained with a rapid maxillary expansion procedure.

Each experimental group consisted of 5 patients, aged between 8 and 12 years wearing the two different appliances. Prior to treatment they were implanted according to the Björk technique. The slow expansion period lasted 7 to 15 weeks followed by a retention period of 12 weeks. Post-retention observations followed 12 weeks after the end of retention.

No significant difference was found between banded and bonded appliances in regard to dental and skeletal expansion and relapse. The amount of skeletal versus dental movements equalled results obtained with rapid maxillary expansion. The relapse tendency appeared lower than with rapid maxillary expansion.

\section{Introduction}

Rapid maxillary expansion (RME) has been performed for many years to widen the upper arch in order to correct unilateral or bilateral crossbites, and to provide sufficient space for the maxillary permanent teeth. During the early 1900 's a great number of investigations led orthodontists to the conclusion that their procedures could influence not only the teeth and the alveolar processes but also the skeleton. Isaacson et al. (1964) reported that forces between 3 and 10 pounds were produced by single activations of jackscrew appliances, while multiple daily activations could result in cumulative loads of 20 pounds or more. The maxilla could then be expanded at a rate of 0.2 to 0.5 $\mathrm{mm}$ per day by opening the midpalatal suture. The concept means that high magnitude forces overcome the skeletal resistance with minimal tooth movement within the alveolar process. Using maxillary implants Krebs (1964) demonstrated that only 50 per cent of the total arch width increase was due to skeletal expansion.

Slow maxillary expansion (SME) procedures using lingual archwires, quadhelix or coil spring types of appliance incorporate a force system of several ounces up to approximately 2 pounds. According to Hicks (1978), 16 to 30 per cent depending on age, of the total expansion obtained by SME is skeletal. The histologic picture of slowly expanded sutural tissue differs significantly from the image obtained by RME. Storey (1973) suggested that the more physiologic sutural adjustments and reparatory reactions produced by SME create a greater sutural stability.

Bonding procedures have been proved to be efficient in replacing the original bands of the fixed appliance. A bonded palatal expander could offer several advantages to a banded appliance (Howe, 1982). It allows parallel movement of the anchor teeth, it is simple to fabricate and can be added at any time during treatment, even with a fully bonded labial appliance in place (Timms, 1980). In addition, the covering of the occlusal surfaces eliminates occlusal interferences during lateral displacement of the bony segments (Spolyar, 1984, Alpern and Yurosko, 1987).

The aim of the present investigation was to 
compare two different types of appliance in the three planes of space: A bonded and banded palatal expander, using a similar continuous light force of 2 pounds (SME). The skeletal and dental linear and angular changes during the active phase of SME, the retention and postretention period will be documented. The ratio between skeletal and dental response to SME will also be evaluated and compared with the results obtained in previous studies on RME.

\section{Subjects and method}

The sample consisted of 10 patients, 6 boys and 4 girls, presenting either a bilateral or a functional unilateral crossbite. They were divided into two experimental groups. In group 1 the age ranged between 8 years 7 months and 10 years 9 months. In group 2 it varied from 8 years 8 months to 12 years. Prior to treatment the patients were implanted according to the Björk (1966) technique at the border of the hard palate and the alveolar process medial to the palatal root of each permanent first molar (M$\mathrm{M}^{\prime}$ ), mesial to the root of the first permanent incisors $\left(\mathrm{I}-\mathrm{I}^{\prime}\right)$, and at the inferior border of each zygomatic process $\left(Z-Z^{\prime}\right)$. They were then documented conventionally.

\section{Appliance design in group 1}

A conventional Minne expander* (Fig. la) was embedded in acrylic. The resin covered the occlusal and labial surfaces of the upper deciduous first and second molars (or first and second premolars) and the permanent first molars. Acidetching was applied on the palatal cusp of the first permanent molars and on the occlusal surfaces of the deciduous molars (or first and second premolars). The appliance was placed using the direct bonding technique with Concise composite and then trimmed until three points of occlusal contact were obtained on each side. On the buccal surfaces of the maxillary first molars, headgear tubes were soldered to a mesh and then bonded in a vertical position (Fig. 1b) to monitor angular changes during the experiment. A threaded wire was inserted in this tube at the time the anteroposterior headfilm was taken. A continuous 2 pounds force coil spring* was used to obtain a maxillary expansion of at least $6 \mathrm{~mm}$ measured between two fine

* Ormco Corporation, Glendora, Ca.

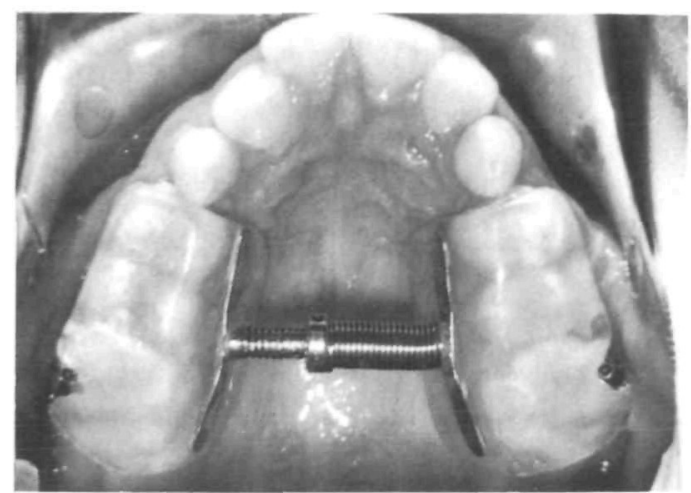

Figure 1(a) Occlusal view of the bonded appliance on patient A.B. The coil spring was totally compressed to produce a continuous force of 2 pounds.

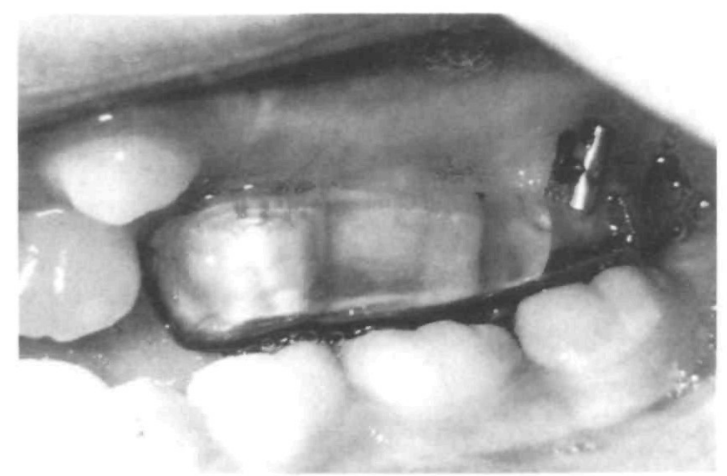

(b) Lateral view of the same appliance on patient N.I. The resin did not contact the headgear tubes, which were bonded secondarily in a vertical direction.

holes perforated in the acrylic over the tip of the mesiolingual cusp of the maxillary first molars. The spring was reactivated every other week.

\section{Appliance design in group 2}

The same Minne expander was soldered in the laboratory to bands previously fitted to the first primary molars (or first permanent premolars) and first permanent molars (fig. 2a). On the buccal side of the permanent molars, headgear tubes were soldered vertically (fig. 2b). After completion of the expansion the fixed appliances were removed. The bonded vertical tube was kept in place in group 1 as well as the maxillary first molar bands in group 2. A conventional maxillary retainer was placed the following day. The patients were instructed to wear it full time for 12 weeks (retention period). They were 


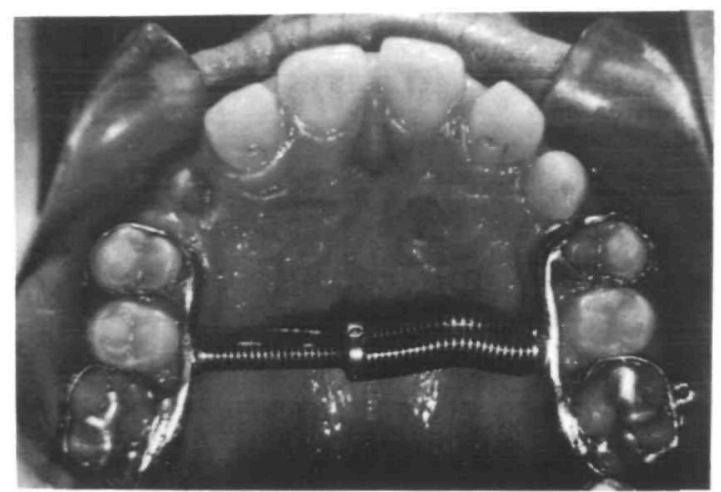

Figure 2(a) Occlusal view of the banded appliance on patient A.S. after completion of the expansion. The coil spring had to be reactivated every two weeks to maintain the same continuous force.

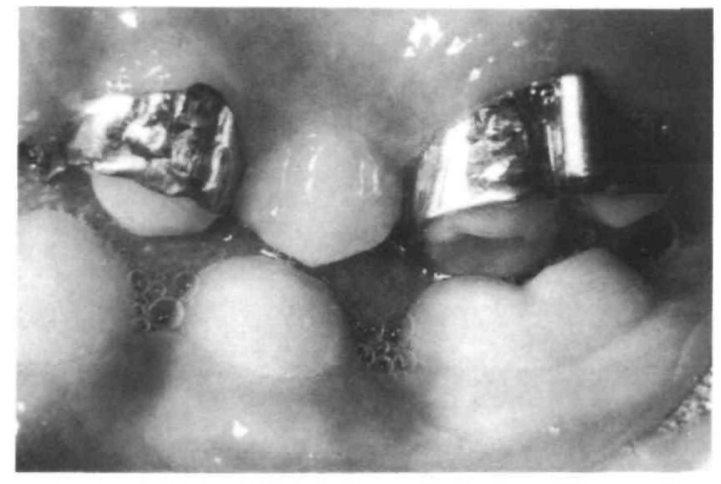

(b) Lateral view showing overcorrection of the crossbite and the vertical headgear tubes soldered to the molar bands.

observed for another 12 weeks without appliances to evaluate the postretention relapse.

\section{Data collection}

Models: Maxillary impressions were taken prior to treatment, at the end of expansion, at the end of the removable retention and at the end of the observation period. For each set of models, a dot was marked with a $3 \mathrm{H}$ pencil on the tip of the deciduous (or permanent) canines and in the centre of the enamel ridge between the distobuccal and the mesiopalatal cusps of the first permanent molars. The intercanine and intermolar distances were recorded twice to the nearest $0.1 \mathrm{~mm}$ with a Boley gauge and averaged.

Radiographs: Occlusal radiographs were obtained before appliance insertion and at the end of expansion to monitor sutural opening. They were examined under the microscope.
Four posteroanterior headfilms were taken, prior to appliance placement, immediately after appliance removal, at the end of the retention period and at the end of the observation period (Fig. 3a). The patients were positioned in a cephalostat with the horizontal and the vertical nasal rest position recorded in order to reorient the patient as closely as possible to the original position. The inter-ear-rods width was also measured.

On the radiograph the intermolar distance was recorded at the intersection between the internal and inferior border of the vertical buccal tube and the labial aspect of the upper first molar. This distance was measured with a Boley gauge to the nearest $0.1 \mathrm{~mm}$ and defined as the overall expansion value.

The interimplant distance was recorded in an identical manner at the centre of the implants. To evaluate the stability of the implants, the distance between the $M-Z$ and the $M^{\prime}-Z^{\prime}$ implants was measured. The skeletal expansion and relapse were calculated as the mean between the interimplant distance variations $\left(\mathrm{I}-\mathrm{I}^{\prime}, \mathrm{Z}-\mathrm{Z}^{\prime}\right.$, $\left.\mathbf{M}-\mathbf{M}^{\prime}\right)$. Changes in angular position of the upper right and left molars and right and left maxilla were analysed according to Hicks (1978) analysis (Fig. 3b).

A cranial reference line (CRL) was drawn by connecting the intersection of the greater wing of the sphenoid bone with the inner cortex of the orbit (latero-orbital point LO). Two molar reference lines (MRL) were constructed along the lateral aspect of the threaded wire, intersecting the CRL. A plane was designed on each side connecting the centre of the zygomatic implant and the implant located on the hard palate (IRL). Three angles (A, B, C) were recorded to monitor the dental tipping and three angles $(D$, E, F) were used to evaluate the skeletal changes. Lateral cephalograms were obtained in a standard position before appliance insertion, immediately after appliance removal and at the end of the observation period to monitor the antero-posterior and vertical changes.

\section{Results}

\section{Clinical findings}

Appliance insertion required more time with the banded Minne expander as separation had to be placed one week prior to band fitting and 

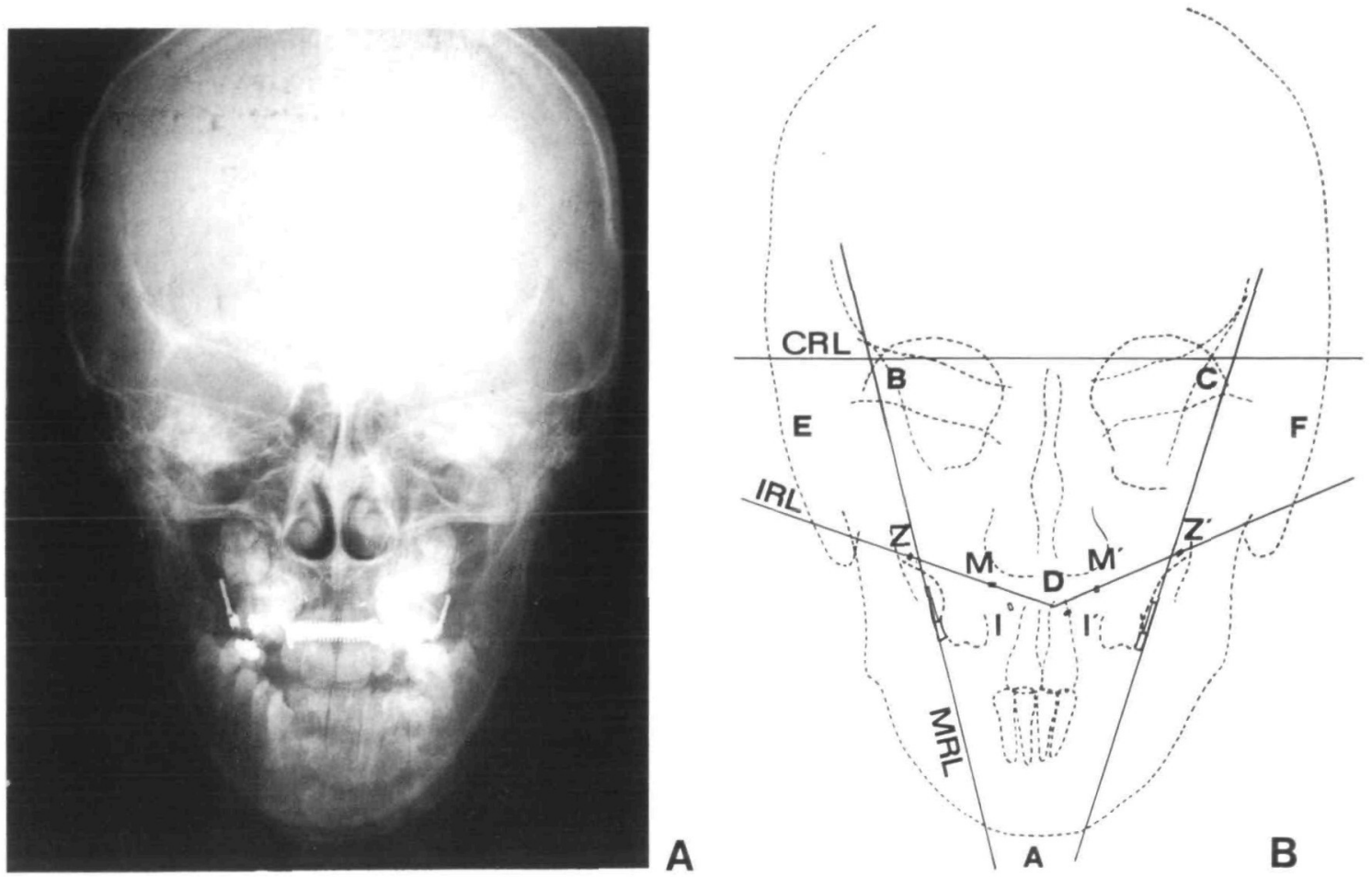

Figure 3(a) Postero-anterior cephalogram from patient S.V.

(b) Reference points, lines and angles measured on the postero-anterior radiographs according to Hicks (1978)

impression. On the other hand, the laboratory work necessary to fabricate the bonded appliance was more sophisticated.

The appliances were very well tolerated by all patients, but hygiene was more difficult to maintain adequately in the bonded expander group. Most of the subjects of both groups developed speech problems during the first few days following appliance insertion. At the end of the expansion period some disturbances remained in group 1. They all disappeared during the retention period of the experiment. In one patient of group 1 (N.I.) the Minne expander had to be removed and replaced by the retainer before completion of the desired expansion because of bond failure on one side. At this point the cast analysis showed an expansion of 6.4 $\mathrm{mm}$ between the molars.

On appliance removal there were significant differences between the two techniques. In group 2 , no more than 15 minutes were required to remove the Minne expander, while 45 minutes to 1 hour were necessary to remove the bonded appliance and all the remaining acrylic material. For these patients the discomfort was also greater and the gingival inflammation more severe. There was a slight depression, corresponding to the removed acrylic, in the palatal mucosa and the tissue was often severely erythematous (fig. 4a). Upon the removal of the banded expander gingival inflammation was also present but to a lesser degree (fig. 4b). However in both groups, the palatal mucosa returned to a normal appearance within one week. During the expansion period none of the patients developed any midline diastema as generally observed in rapid maxillary expansion procedures.

\section{Cast analysis}

Table 1 describes the expansion gained between the canines and the molars and the total relapse calculated during the retention and postretention periods. In all patients of the two groups the intermolar distance increased more than the intercanine distance. The mean width increase between the canines was $6.4 \mathrm{~mm}$ in group 1 and 


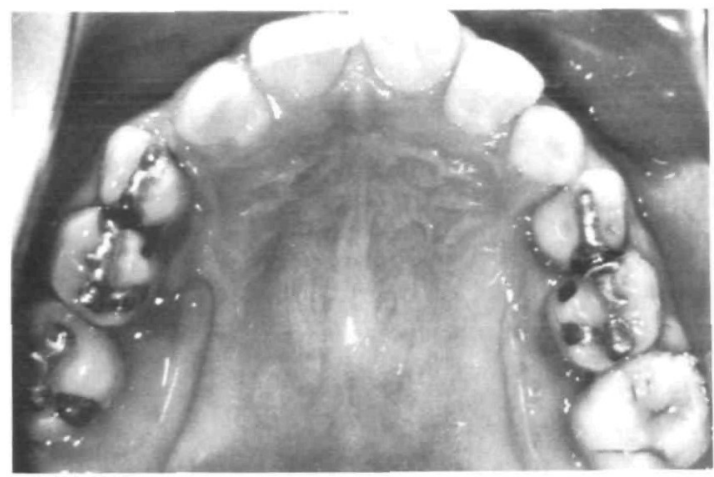

Figure 4(a) Patient A.L.: Occlusal view immediately after bonded appliance removal. No midline diastema can be observed. A severe gingival inflammation surrounded the teeth previously embedded in acrylic.

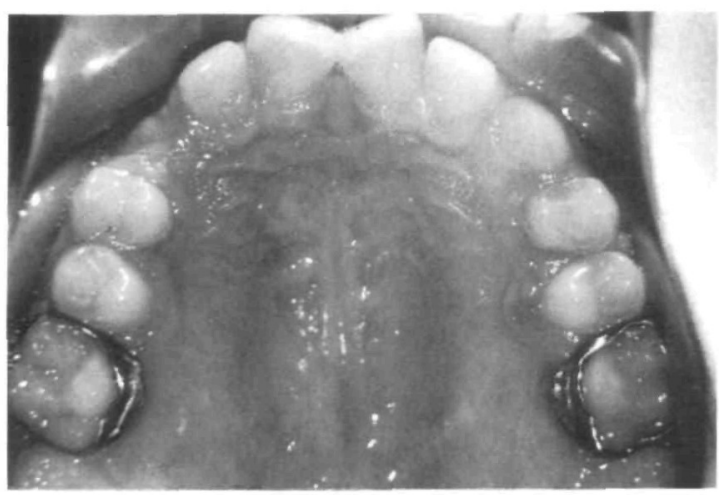

(b) Patient Y.G.: after removal of the banded expander, the palatal mucosa showed less erythema.

$5.3 \mathrm{~mm}$ in group 2. The intermolar distance increased $7.9 \mathrm{~mm}$ in group 1 and $8.3 \mathrm{~mm}$ in group 2 . The relapse which occurred during the retention (12 weeks) and postretention period (12 weeks) followed the same pattern in both groups. The mean relapse of the intercanine expansion was $1.6 \mathrm{~mm}(25 \%)$ in group 1, and $1.2 \mathrm{~mm}(22 \%)$ in group 2 . The intermolar relapse was $2.3 \mathrm{~mm} \mathrm{(29 \% )} \mathrm{in} \mathrm{group} \mathrm{1,} \mathrm{and} 2.5 \mathrm{~mm} \mathrm{(30 \% )}$ in group 2.

\section{Radiographic findings}

Occlusal radiographs. In all the patients an opening of the midpalatal suture was observed on the radiograph taken at the end of the expansion period. The sutural widening appeared larger anteriorly. Minimal bone separation was found in the second molar region. Although no radiolucency could be demonstrated radiographically along the suture (Fig. 5a) as generally observed in rapid maxillary expension (Fig. 5b) there would appear to be additional bone deposition within the suture, since the distance between the implants increased.

Posteroanterior radiographs. Linear changes: Table 2 shows the overall expansion and relapse measured between the buccal tubes of the first permanent molars, and the skeletal expansion and relapse which occurred in the subjects of group 1 and group 2. The mean net overall expansion as well as the mean net overall relapse were almost identical in both groups. The skeletal expansion was slightly greater for the patients wearing a bonded appliance, but then the skeletal relapse was also greater in this group. The contribution of the skeletal expansion to the total transverse change amounted to 56 per cent in group 1 (mean) and 51 per cent in group 2 (Fig. 6). Twenty-seven per cent of the overall expansion gained in group 1 was lost and more than half was due to skeletal relapse $(61 \%)$. In group 2 an almost identical relapse was observed $(29 \%)$ but then only 32 per cent of the

Table 1 Expansion and relapse recorded on casts of group 1 and group $2(\mathrm{~mm})$

\begin{tabular}{|c|c|c|c|c|c|}
\hline & \multirow[b]{2}{*}{ Age } & \multicolumn{2}{|l|}{ Intercanine } & \multicolumn{2}{|l|}{ Inter molar } \\
\hline & & Expansion & Relapse & Expansion & Relapse \\
\hline $\begin{array}{l}\text { Group I } \\
\bar{x} \\
\text { s.d. }\end{array}$ & 10.1 & $\begin{array}{l}6.4 \\
1.1\end{array}$ & $\begin{array}{l}1.6 \\
0.5\end{array}$ & $\begin{array}{l}7.9 \\
1.5\end{array}$ & $\begin{array}{l}2.3 \\
0.8\end{array}$ \\
\hline $\begin{array}{l}\text { Group II } \\
\bar{x} \\
\text { s.d. }\end{array}$ & 10.5 & $\begin{array}{l}5.3 \\
1.9\end{array}$ & $\begin{array}{l}1.2 \\
0.3\end{array}$ & $\begin{array}{l}5.3 \\
1.1\end{array}$ & $\begin{array}{l}2.5 \\
0.6\end{array}$ \\
\hline
\end{tabular}




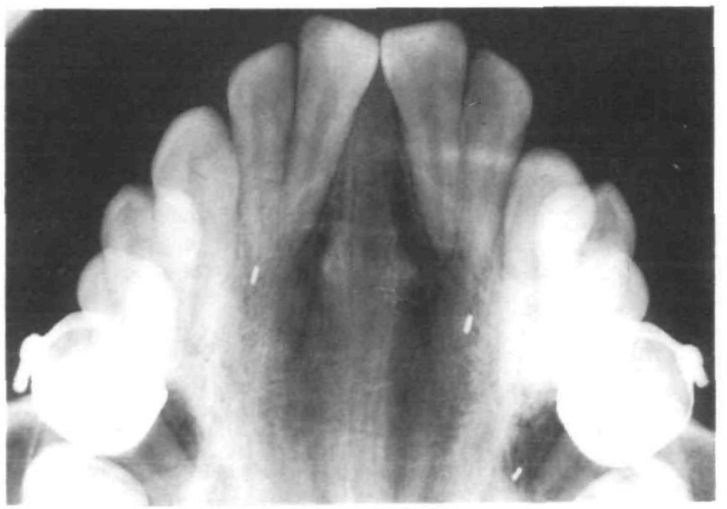

A

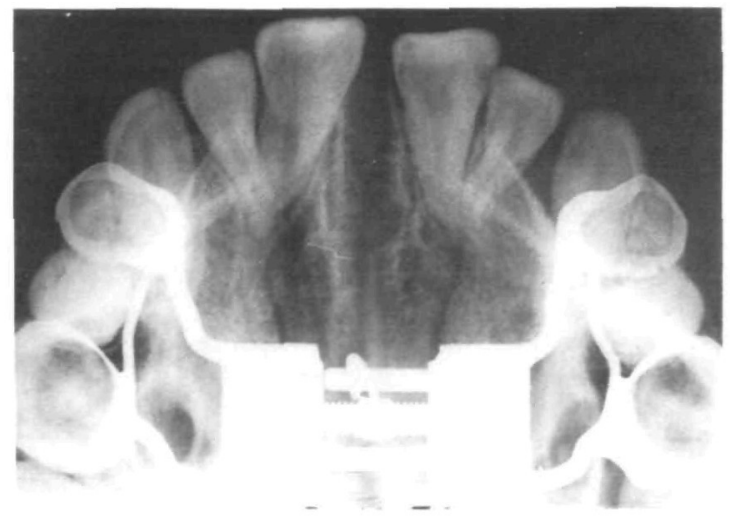

B

Figure 5(a) Patient I.G.: Occlusal radiograph taken immediately after appliance removal (end of expansion), showing a sutural opening. No radiotransparency can be found along the suture which indicates new bone deposition.

(b) Occlusal radiograph taken on a patient who was not included in this study and presented as a comparison. At the end of a rapid maxillary expansion a radio-transparency is clearly seen along the midpalatal suture' as well as a midline diastema.

Table 2 Expansion and relapse recorded on P.A. radiographs of group 1 and group $2(\mathrm{~mm})$.

\begin{tabular}{|c|c|c|c|c|c|c|}
\hline & \multicolumn{2}{|l|}{ Overall } & \multirow[b]{2}{*}{ Total relapse } & \multicolumn{2}{|l|}{ Skeletal } & \multirow[b]{2}{*}{ Total relapse } \\
\hline & Expansion & $\begin{array}{l}\text { Relapse during } \\
\text { retention }\end{array}$ & & Expansion & $\begin{array}{l}\text { Relapse during } \\
\text { retention }\end{array}$ & \\
\hline \multicolumn{7}{|c|}{ Group 1} \\
\hline$\overline{\mathrm{x}}$ & 7.9 & 0.5 & 2.1 & 4.4 & 1.1 & 1.3 \\
\hline s.d. & 1.9 & 0.2 & 0.8 & 1.2 & 0.3 & 0.4 \\
\hline \multicolumn{7}{|c|}{ Group 2} \\
\hline$\overline{\mathrm{x}}$ & 7.7 & 0.7 & 2.3 & 3.9 & 0.4 & 0.7 \\
\hline s.d. & 1.8 & 0.4 & 0.4 & 0.9 & 0.3 & 0.4 \\
\hline
\end{tabular}

overall relapse could be attributed to the skeletal component.

Angular changes: For the purpose of clarifying the results, only the dental angle $A$, which relates the right and left upper first molars to each other and the skeletal angle $D$ which relates the right and left maxillae to each other are reported. During the expansion period the dental angle $\mathrm{A}$ decreased $9.8^{\circ}$ (mean) in group 1 and $6.5^{\circ}$ in group 2, indicating dental tipping (Fig. 7). It returned $6.8^{\circ}$ towards its original angulation during the retention and postretention period in group 1 and $5.3^{\circ}$ in group 2. The skeletal angle $\mathrm{D}$ decreased $5.3^{\circ}$ (mean) in group 1 , and $6.8^{\circ}$ in group 2 during the expansion phase (Fig. 8) indicating a lateral rotation of the two maxillary bones. A very slight relapse was observed during the retention and postretention periods $\left(+1^{\circ}\right.$ in group 1 , and $+0.8^{\circ}$ in group 2). The skeletal and dental tipping were characterized by a great individual variability.

Lateral cephalograms: Superimpositions of the lateral headfilms taken before and after the expansion and at the end of the observation period, as well as the determination of the angles SNA, ANB, SN-MGo' SN-ANS/PNS and ANS/PNS-MGo' revealed no significant change during the experiment in all the patients. There was also no significant difference between the bonded and the banded expander groups. During the expansion period the SNA angle increased $1^{\circ}$ (mean), and the ANB angle $0.5^{\circ}$ in both groups. The SNA and ANB angles decreased $0.5^{\circ}$ during the retention and postretention phases. The tendency towards an opening of the mandibular plane angle, and a decrease 


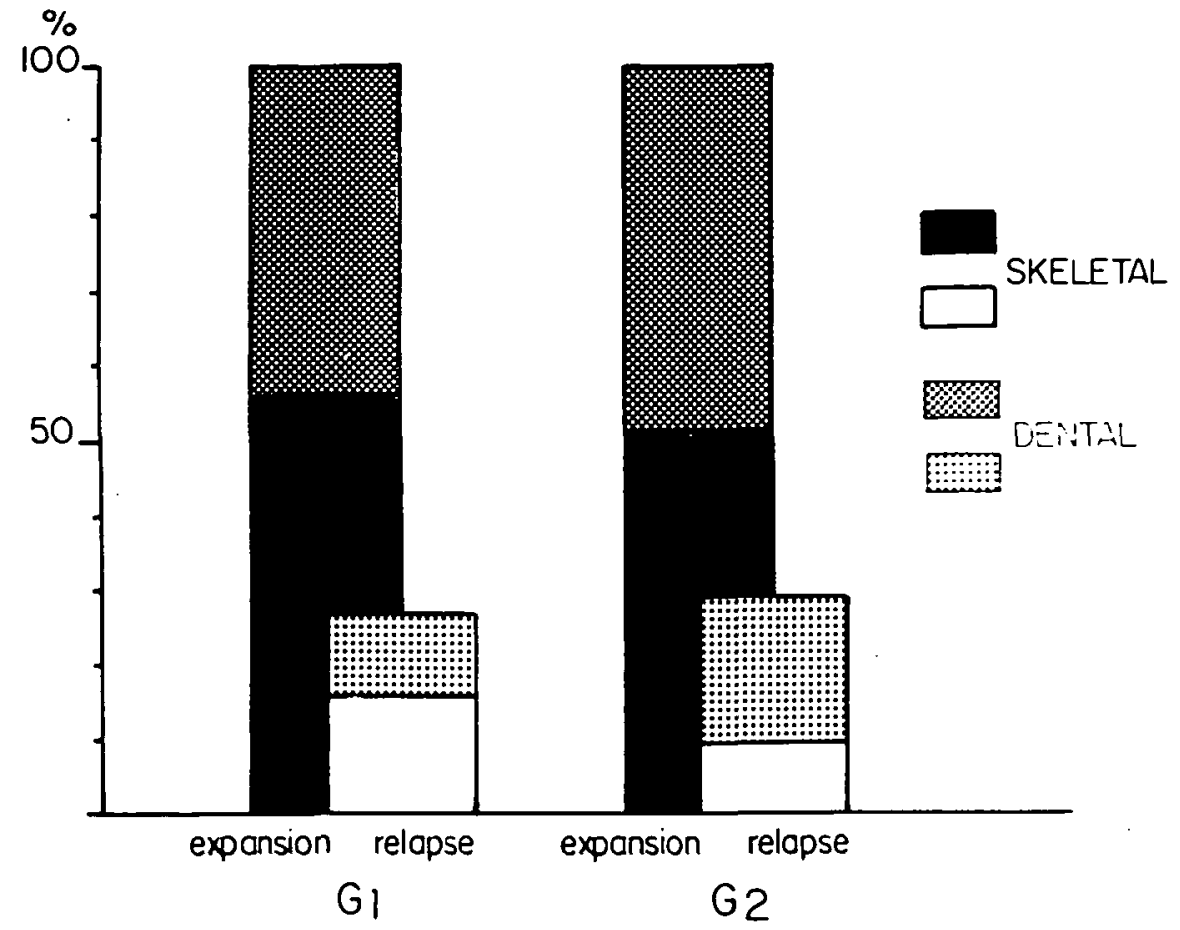

Figure 6 Histogram representing the overall, the dental and the skeletal expansion and relapse in the two groups.

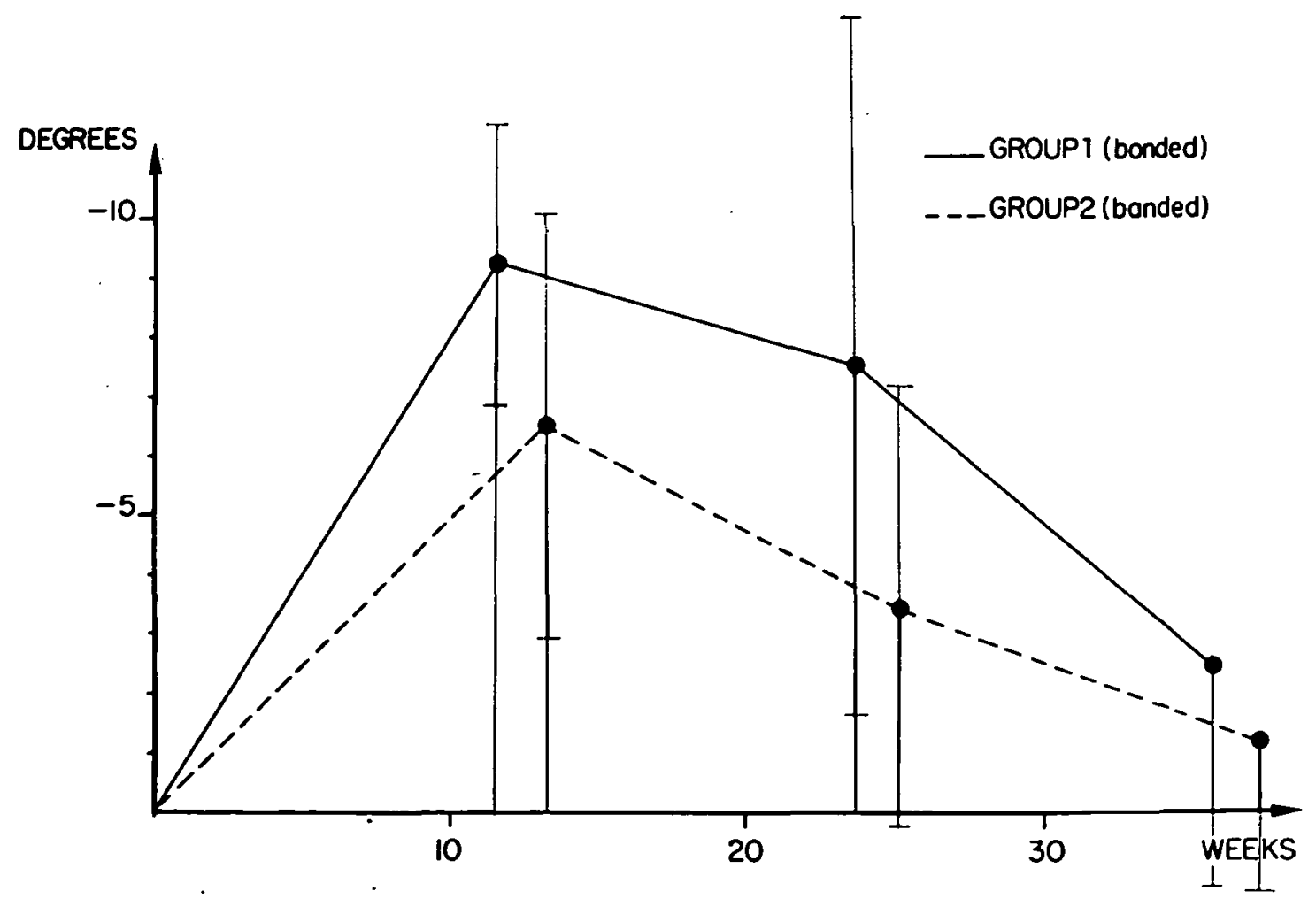

Figure 7 Histogram showing the mean dental angular changes (measured by the angle $\mathrm{A}$ ), during the expansion, the retention and the post retention period; in the two:groups. 


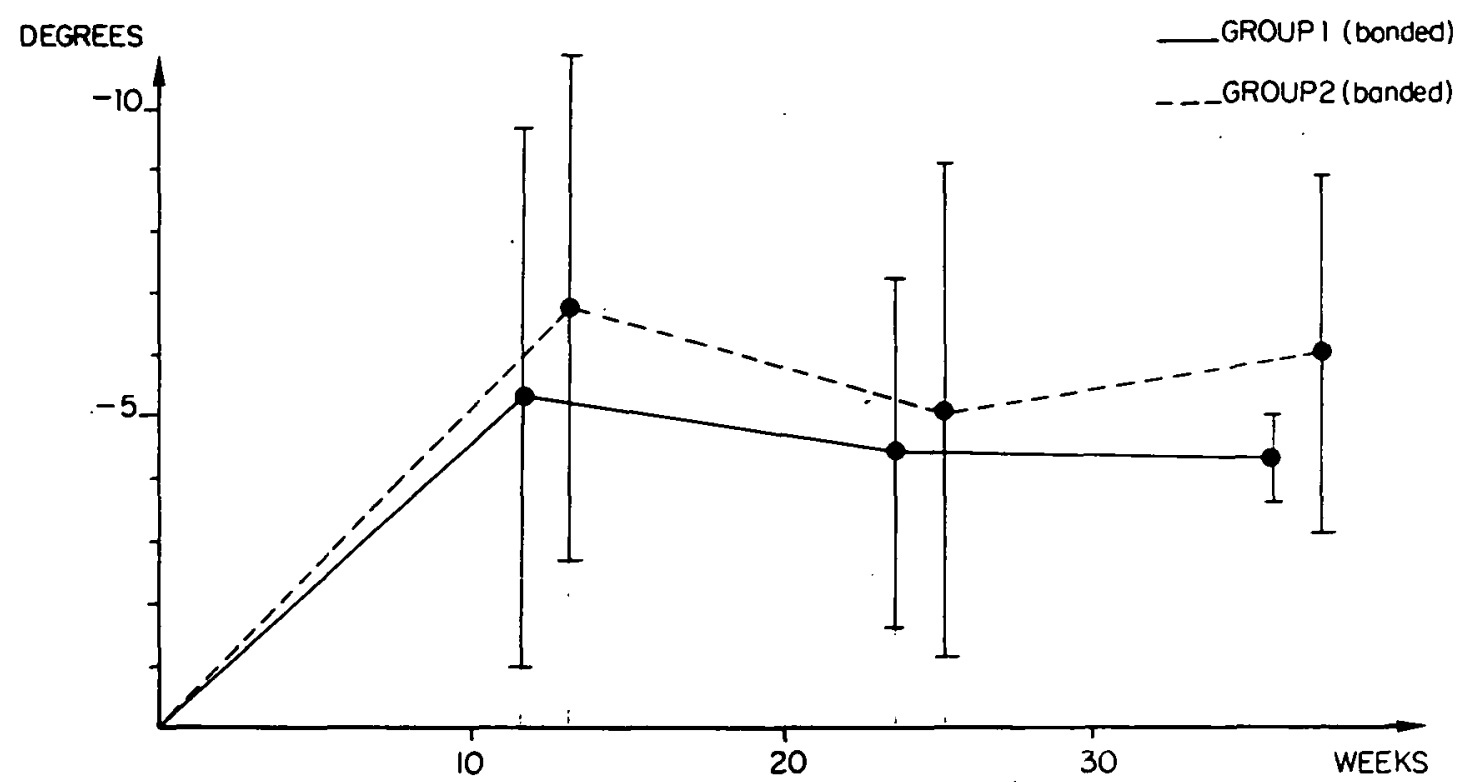

Figure 8 Histogram showing the mean skeletal angular changes (measured by the angle D) during the expansion, the retention and the postretention period in the two groups.

of the SN-ANS/PNS angle was generally observed during the expansion phase, however none of the values varied more than $2^{\circ}$ from the original in the two groups. These values returned towards their initial readings during the post expansion period.

\section{Discussion}

Various types of device have been used to expand the maxilla by separation of its two halves. With the development of bonding procedures, new appliance designs have been reported (Howe, 1982; Spolyar, 1984; Alpern and Yurosko, 1987). However, insufficient data are available to compare their effects to the original banded expander. The findings of the present experiment have demonstrated that no significant difference could be detected in the amount of maxillary expansion, the percentage of skeletal response and the degree of dental tipping between banded and bonded appliances. In the two experimental groups the skeletal expansion amounted to approximately $50 \%$ of the total arch width expansion. Using the same amount of force, Hicks (1978) obtained only $28 \%$ of skeletal expansion. The differences could be explained by the less rigid appliance used in his study and by the variations in patients' ages (10 to 15 years).

Castro (1979) applied a continuous 2 pound load with a rigid fixed expander on patients aged between 8 years 7 months and 13 years. He measured 40 per cent of skeletal expansion.

During the post expansion period the same type of retainer was inserted in the two groups and was worn for 12 weeks. An identical amount of overall relapse was observed during this phase, but the skeletal relapse appeared slightly greater in the bonded expander group. At the end of the observation period the total relapse was approximately 30 per cent of the total arch width expansion.

As the two parts of the maxilla separate from each other, they also tip buccally with a centre of rotation located approximately at the frontomaxillary suture (Haas, 1961, 1970; Werz, 1970). The results of the present study confirmed these findings and showed that asymmetrical angular changes occurred in both groups. The buccal molar tipping could be explained by the more occlusally transmitted force from the coil spring to the acrylic covering the occlusal surfaces of the anchor teeth. The change in molar angulation is not only due to dental tipping at sutures but 
also an effect of alveolar bending. In the two groups the change in angular position of the two alveolar processes ranged from $5^{\circ}$ to $6^{\circ}$. These changes remained stable throughout the retention and postretention periods. The removable maxillary plate used as a retention device might be responsible for this stability. The slow rate of expansion could also play a major role in obtaining the permanent skeletal change.

On the other hand, the molars tipped back during the retention and the postretention periods to their original angulation. It can then be concluded that following expansion, tooth movement occurs within the alveolar bone as previously mentioned by Cotton (1978), who postulated that the post expansion molar angular changes were related to the stretched fibres of the attached palatal mucosa. By performing incisions along the palate $3 \mathrm{~mm}$ away from the teeth, Muguerza and Shapiro (1980) attempted without success to relieve the stretch of the mucoperiosteum after SME and to reduce the overall relapse. Haas (1961) reported forward and downward movement of the maxilla with RME. These findings were not totally supported by Werz and Dreskin (1977) who also observed a downward and slightly forward maxillary displacement during the suture opening. However a full recovery usually occurred during stabilization. The cephalometric analysis of the two experimental groups in this study was in agreement with this last observation.

Approximately 3 months were required to obtain $8 \mathrm{~mm}$ of maxillary widening. The rate of expansion was $0.7 \mathrm{~mm}$ per week in the bonded group and $0.6 \mathrm{~mm}$ per week in the banded group. This difference cannot be considered significant as a great variability was observed between the individuals. In RME the rate usually varies from $0.2 \mathrm{~mm}$ to $0.5 \mathrm{~mm}$ per day. An increase of 8 to $10 \mathrm{~mm}$ of arch width can be gained within one month. The supporters of rapid palatal splitting believe that minimal tooth movement and maximal skeletal displacement are produced by high magnitude forces. Krebs (1958) used metallic implants on four patients aged 8 years 7 months to 13 years. RME in his experiment produced only $47 \%$ of skeletal expansion, which is not significantly different from our present findings after SME. A continuous force of two pounds can produce an orthopaedic effect on all the maxillary complex and its surrounding structures. Using laser holography
Pavlin and Vuvicevik (1984) confirmed this statement.

A tendency towards a decrease in the width of the dental arches during the 4 to 5 years following RME was reported by Krebs (1964). Linder-Aronson and Lindgren (1979) recorded the results of RME 5 years postretention. Only $45 \%$ of the initially achieved expansion was maintained. These findings are in agreement with the observations of Stockfish (1969) who found in his sample $50 \%$ of relapse within 3 to 5 years post retention. Hicks (1978) stipulated that only 8 weeks of fixed retention were required following SME. He did not recommend removable retainers. In the present investigation removable retainers were inserted the day following the end of expansion and kept for 12 weeks. The decrease in arch width was monitored only for 12 weeks. The correction of the crossbite was maintained in all subjects but an average of $30 \%$ of relapse was found. No conclusion can yet be drawn on the final stability of the achieved expansion. Records will be gathered in the future to establish a significant comparison with RME. Nevertheless the skeletal expansion appeared reasonably stable during the post-expansion phase. This can be attributed to the quality of the new bone deposition along the midpalatal suture. Ten Cate et al. (1977) observed an initial inflammatory stage RME suggesting injury. It is followed by a proliferative repair phenomenon consisting of osteogenesis and fibrogenesis and finally by remodelling and regeneration of the suture. Ekström et al. (1977) found a rapid increase of mineral content within the suture during the first month following the completion of rapid maxillary suture opening. In contrast with RME, during and after SME none of the patients developed a midline diastema. The relatively low magnitude of force probably did not overcome the strength of the transeptal fibers which held the crowns together.

Root resorption on the buccal surface of abutment teeth secondary to RME have been described by Langford and Sims (1982), who also observed repair of the defect by cellular cementum exclusively. Future studies will be necessary to compare the anchor teeth root response to SME. The periodontal condition of the maxillary teeth examined 12 weeks following retention appeared excellent in the two groups of the present study. These observations confirmed Greenbaum and Zachrisson's (1982) findings. 
They compared the periodontal status of a group of patients after RME, after SME and of a control group. No significant difference could be determined.

On the basis of these findings, the following conclusions can be made:

1. The skeletal and dental response to SME is identical whether a bonded or a banded appliance is used.

2. Slow maxillary expansion performed with a continuous force of 2 pounds can produce orthopaedic effects on the maxilla and its surrounding structures that are comparable to those obtained by a rapid maxillary expansion using heavy forces.

3. The slow rate of expansion might be responsible for the postexpansion stability. This hypothesis remains to be confirmed by long term data.

\section{Address for correspondence}

$\mathrm{K}$ and $\mathrm{C}$ Mossaz, Section de Médecine Dentaire, 19 , rue Barthélemy-Menn, 1211 Geneva 4, Switzerland

\section{References}

Alpern M C, Yurosko J J 1987 Rapid palatal expansion in adults with and without surgery. The Angle Orthodontist 57: $245-263$

Björk A 1966 Sutural growth of the upper face studied by the implant method. Acta Odontologica Scandinavia 24: 109-127

Castro C M 1979 Slow maxilla expansion: a clinical study of the skeletal and dental response during and following the application of a low magnitude force (Master's Thesis) University of Washington Seattle

Cotton L A 1978 Slow maxillary expansion: skeletal versus dental response to low magnitude force in Macaca mulotta. American Journal of Orthodontics 73: 1-23

Ekström C, Henrichson C O, Jensen R 1977 Mineralization in the midpalatal suture after orthodontic expansion. American Journal of Orthodontics 71: 449-455

Greenbaum K R, Zachrisson B V 1982 The effect of palatal expansion therapy on the periodontal supporting tissues. American Journal of Orthodontics 81: 12-21

Haas A J 1961 Rapid expansion of the maxillary dental arch and nasal cavity by opening the midpalatal suture. The Angle Orthodontist 47: 186-192
Haas A J 1970 Just the beginning of dentofacial orthopaedics. American Journal of Orthodontics 57: 219-255

Hicks E P 1978 Slow maxillary expansion: a clinical study of the skeletal vs dental response to low magnitude force. American Journal of Orthodontics 73: 121-141

Howe R P 1982 Palatal expansion using a bonded appliance. American Journal of Orthodontics 82: 464-468

Isaacson R J, Wood J L, Ingram A H 1964 Forces produced by rapid maxillary expansion. The Angle Orthodontist 34: $256-270$

Isaacson R J, Ingram A N 1965 Forces produced by rapid maxillary expansion. III. Forces present during retention. The Angle Orthodontist 35: 178-186

Krebs A 1958 Expansion of the midpalatal suture studied by means of metalic implants. European Orthodontic Society Report 34: 163-171

Krebs A 1964 Rapid expansion of midpalatal suture by fixed appliance. An implant study over a 7 year period. Transactions of European Orthodontic Society: 141-142

Langford S R, Sims M R 1982 Root surface resorption, repair and periodontal attachment following rapid maxillary expansion in man. American Journal of Orthodontics 81: $108-115$

Linder-Aronson S, Lindgren J 1979 The skeletal and dental effects of rapid maxillary expansion. British Journal of Orthodontics 6: 25-29

Muguerza O E, Shapiro P A 1980 Palatal mucoperiostomy: an attempt to reduce relapse after slow maxillary expansion. American Journal of Orthodontics 78: 548-558

Pavlin D, Vuvicevik P 1984 Mechanical reaction of facial skeleton to maxillary expansion determined by laser holography. American Journal of Orthodontics 85: 498 507

Spolyar J L 1984 The design, fabrication and use of a fullcoverage bonded rapid maxillary expansion appliance. American Journal of Orthodontics 86: 136-145

Stockfisch H 1969 Rapid expansion of the maxilla: success and relapse. Transactions of European Orthodontic Society $469-481$

Storey E 1973 Tissue reponse to the movement of bone. American Journal of Orthodontics 64: 229-247

Ten Cate A R, Freeman E, Dickinson J B 1977 Sutural development: structure and its response to rapid expansion. American Journal of Orthodontics 71: 622-636

Timms D J 1980 A study of basal movement with rapid maxillary expansion. American Journal of Orthodontics 77: $500-507$

Werz R A 1970 Skeletal and dental changes accompanying rapid midpalatal suture opening. American Journal of Orthodontics 58: 41-66

Werz R A, Dreskin M 1977 Midpalatal suture opening: A normative study. American Journal of Orthodontic 71: $367-381$ 